\title{
Interactive Data Mining: A Brief Survey
}

\author{
M. Manohara, R. Dinesh, and S. M. Sowmya
}

\begin{abstract}
In the rapidly growing technologies, there exist large amount of data generated, captured and maintained in many industries. There is a need to analyse, and take strategic decision based on the historical data and to update the existing data as per the current need. In this paper, we have conducted a comprehensive survey on the areas related data mining such as Data Extraction, Transformation and Loading; Interactive Data mining; Knowledge Discovery and Content summarization. This survey highlights the challenges of the existing methods, evolutionary growth of technologies. Towards the end, the paper highlights prospective avenues for further research in the area of Interactive Data Mining.
\end{abstract}

Index Terms-Data visualization, data summarization, knowledge discovery in databases(KDD).

\section{INTRODUCTION}

\section{A. Data Mining}

Data mining, which is also known as Knowledge Discovery in Databases is a field at the intersection of computer science and statistics is the process that attempts to discover patterns in large data sets. It utilizes methods at the intersection of artificial intelligence, machine learning, statistics, and database systems. The overall goal of the data mining process is to extract information from a data set and transform it into an understandable structure for further use.

\section{B. Data Visualization}

The data visualization techniques can be used in the pre-processing stage (e.g., initial data analysis and feature selection) and the post processing stage (e.g., visualization of results, tests of performance of classifiers, etc.). Visualization may support the analysis of the classifier and thus increase the comprehensibility of discovered relationships.

\section{Data Summarization}

For data pre-processing to be successful, it is essential to have an overall picture of your data. Descriptive data summarization techniques can be used to identify the typical properties of your data and highlight which data values should be treated as noise or outliers. Descriptive data summarization provides the analytical foundation for data pre-processing.

The basic statistical measures for data summarization include mean, weighted mean, median, and mode for measuring the central tendency of data, and range, quartiles,

Manuscript received February 4, 2013; revised April 15, 2013.

The authors are with School of Engineering and Technology, Jain University, Bangalore (e-mail: kotamanu@gmail.com, dr.dineshr@gmail.com,mssowmya.sbmjce@gmail.com). interquartile range, variance, and standard deviation for measuring the dispersion of data. Graphical representations, such as histograms, boxplots, quantile plots, quantile-quantile plots, scatter plots, and scatter-plot matrices, facilitate visual inspection of the data and are thus useful for data pre-processing and mining.

\section{RELATED WORK}

In today's world, data mining or KDD [1]-[5] is a very important task for the database designer, business men and many other people. Before the task of data mining, the data has to be collected from various sources. Once the collected data is stored in the database, it has to be analysed to extract the different hidden information. Subsequently the hidden facts are extracted and the conclusion or result has to be displayed to the user in the required form.

The result displayed using natural language will be difficult for the end user to understand or to analyse it because, either result will be ambiguous or they don't have required time to read it. Thus the results need to be displayed in the required format depending on the user need. Hence, data visualization techniques also play the key role in data mining applications. In data summarization user wants the whole result in one page or in minimum number of pages; so, the display format for the result has to be decided. On the other hand, if the user wants more information about the result then link must be provided so that user will get more information about the result that is displayed, i.e. the result and the other detailed information should be in hierarchical fashion. The detailed information shall give the user an idea on what basis the result has to be analysed.

In India, particularly in government sectors, large amount of important data is still available in paper format, because of this, analysis or decision making takes longer time. If all these data are made available in electronic form in database, then the data mining techniques and knowledge discovery techniques can be applied to extract the hidden facts which will be useful for making faster decision which helps in the development of the country.

There are many issues in implementing this: primarily, it has to be decided that how to convert and store the data that is in the form of paper document, where the content are either in hand written or printed format in a database. It is seldom possible to manually enter the data, hence it takes years to create the database and do the analysis. If the researcher implements the above mentioned concept, it definitely will save lot of effort and money and the people can save their valuable time by using this information. It will also help them to take correct decision in their business.

Data summarization purely depends on what type of the data is present in the database, that is, text, audio, images or 
combination of all these. Different summarization techniques can be used for different types of data. In this research, we propose to develop a user interactive data mining and data summarization technique i.e. whatever form of result the user wants is given as input to the system which is then analysed by it and the result is produced in the required format.

During the last two decade, many attempts have been made on development of ETL (Extract Transform and Load), data mining and visualization techniques on large data set. [6] Have proposed modified HRU (Harrrison-Ruzzo-Ullman) algorithm constructing the data models to select required pattern from the spatial data using multidimensional cube of various groups. In [6], previous HRU algorithm has not been used due to its restriction in the cuboids selection. However, they have proposed extension to the previous HRU by considering the parameters like total space and frequency of the cuboids being queried. Greedy techniques have been used for cuboids materialization. The main aim of their research is to generate efficient multidimensional cube for spatial and non spatial data of the climate. However, they have not applied any data mining techniques for decision making. The work [7] describes, how the data mining techniques can be applied to government GIS (geographic information system) data. The method mainly aims at handling the uncertainties arise due to attributes. However, it does not consider the uncertainties caused due to scaling, resolution and sampling. The method was applied to study the land coverage and utilization in certain regions of guizhou province, southwest china. The entire region under study was divided into three regions viz., Lush Land, Green Land and Crop Land. The main limitation of the method is that it handle only one type of uncertainty, also the method has not been compared with the other existing methods to establish the superiority of their results. [8] Have proposed the data summarization quality check based on the criterias imperative, minimum coverage, minimum relevance, succinctness and usefulness and also about the data summarization using the concepts of hierarchies both for the data sets and fuzzy data set. Minimum coverage criteria are applied for the fuzzy data summarization and for hierarchical summarization uses tree data structure. In fuzzy data sets cluster is constructed based on [9] method. For the purpose of testing, they have considered restaurant data. But the same techniques might not give the same performance with the other data set. [10] Have proposed a method for compressed data summarization. They compress entire dataset before applying data mining approaches. However, the overall compression of entire dataset is time consuming, this could have been avoided by compressing only the relevant attributes, also the other limitations due to compression techniques are still exist with this method. [11] Have proposed a data mining techniques for video data for game of cricket, to analyse the playing pattern and its outcomes. The principal component analysis (PCA) has been applied to study the frequent patterns and their relationship between the attributes. Subsequently, they are able derive the association rules for the playing pattern of the player (e.g., Sachin Tendulkar). Their method was able to achieve the overall reduction in data size by $18 \%$. [12] Have discussed about the intelligent query answering for the data bases. Depending on the datasets constructed knowledge rich database model to answer the interactive query. One of the components of the data model uses KDT (knowledge discovery tools) for interactive query management. The query answering mechanism has been categorised into four classes: 1) data query and direct answering, 2) data query and intelligence answering, 3) knowledge query and direct answering and 4) knowledge query and intelligent answering. They have used university database for the testing purpose. [13] Aims to find the relationship between the different attributes in the semi structured data set; as the XML uses association rule algorithm "Apriori" and "Frequent Pattern Growth" (FP growth) algorithms which are implemented in java. Most of the earlier researches used to store the XML in relational database before apply the association rules, this involves the manual work. During this research period Xquery was available to construct the association rule using Apriori algorithm for XML data. In this research, the association rule is constructed directly on the XML documents and XML repository, the result is compared with the Xquery result and, the researcher will be able to prove that their FP Growth algorithm is better than the Xquery Apriori algorithm. But the main limitation of the research is that it works only on semi structured data like XML. [14] Discusses about the problems with the high dimensional data sets and, also provide the solution to select the sub groups from the datasets and derives the relationships between subgroups. It is shown that if PCA used for the selection of the subgroups, it is not able get the relationships between the subgroups, since PCA is a global approach trying to identify the maximum separation of data entities globally. For the model building the study uses factor analysis in statistics and feature selection in machine learning methods. Pearson correlation is used to determine the correlation between the attributes. When the user select the datasets, immediately locally for the datasets PCA, it will be applied to find the relationship between the selected datasets attributes. Data selection and its relationships can be calculated interactively.

Interactive data mining is not a new concept, especially when data mining is perceived from the statistical point of view [15]. To select an optimal learning algorithm for a certain task, two popular mechanisms exists: 1) one approach is to learn a decision tree for the applicability of the available algorithms based on the data characteristics [16] and 2) another approach is a user-centred mechanism used in the consult part of the MTL-Project [17]. The survey [18], which dealt with the question of how companies can apply inductive learning techniques, concluded that the process of machine learning should primarily be user driven, instead of data or technology driven. Such a conclusion can also be found among many of the other papers [19], [20] Have proposed a graphical interactive approach to machine learning that makes the learning process explicit by visualizing the data and letting the user "draw" decision boundaries in a simple but flexible manner. A similar research effort can be found in [21]. However, these visualization techniques could make data mining more intuitive, it may decrease the mining efficiency in handling realistic problems where data mining could be very complicated and involve different mining mechanisms. To address this problem, [22] proposed an approach which involves systematically decomposing a data 
mining process into subtask and selecting appropriate problem solving methods and algorithms. [23] Proposed online interactive data mining for student database. [24] Have used KDT tool is for intelligent query answering (IQA) In this method, generalization is done for all the generalized attributes before run time, while in the previous methods generalization is done during the run time. Desirable threshold is used to control the hierarchical levels. Earlier researches used discovery mechanisms to define attributes missing in one information system by mining other information systems. This study uses a knowledge-rich data model and the knowledge discovery tools to discover generalized rules. A limitation of their approach is that there is no mechanism to deal with changes to databases. [25] Reviews the already available interactive data mining approaches, which provides, the best data mining techniques for the given dataset and mined pattern is displayed to the user using various visualization techniques. User can select the pattern and the mining techniques of his/her interest. Almost all the researches provides interface to select the classifiers, clusters or the association rule of users choice. [26] Discusses about the available automatic article content summarization accurately and comprehensively. According to this study, automatic content summarization is classified into five categories: automatic extraction, understanding-based automatic summarization, information extraction, automatic summarization based on discourse and, automatic summarization based on user-query. From the study, they concluded that, automatic summarization based on discourse is better than the other four methods. ROUGE (Recall-Oriented Understudy for Gisting Evaluation) is an automatic summarization evaluation package and, it is shown that they are able to get the promising result. Automatic content summarization technique works well with the article content summarization but might not work efficiently and effectively with the large data sets. [27] Presents a summarizer system, named iWIN (information on the Web In a Nutshell), that is able to perform an automatic summarization of multiple documents through a semantic analysis of the text. The study uses a ranking method to evaluate the relevance of the information for the specific user, a clustering method based on the document representation in terms of set of triplets (subject, verb, object) and, a sentences selection/ordering process to make the final summary as much readable as possible. Their result shows that the ROUGE has superior performance.

\section{DISCUSSION}

From the above discussion, it is clear that most of the existing approaches deal with solving a specific problem (application) for specific data set. There exists no generic rule/technique which works for all data sets in all possible applications. Hence, the major focus of the current research in the area related to data mining is towards building generic framework which can be used across the applications.

It is also clear from above mentioned discussion that, though many techniques exist in solving various data mining issues. None of the techniques fully addresses all the issues pertaining to data mining. Hence, the proposed research work focuses on addressing the following major challenges.

With the recent advances in Big Data, conduction of research in coming out with generic approaches for data mining is very interesting and also important aspects. By coming out with generic framework for data mining most of the issues pertaining to Big Data analytics can be solved.

\section{CONCLUSION}

Research in the area of Interactive Data Mining has been presented in this paper. The paper highlighted the aspects and challenges in the areas related to data mining such as ETL, Interactive Data Mining, Knowledge Discovery and Content Summarization. Also we have presented the prospective avenues for further research in the area of data mining and its relevance to Big Data.

\section{REFERENCES}

[1] R. Agrawal and R. Srikant, "Fast Algorithms for mining association rules," in Proc. 20th International Conference on VLDB, Santiago, 1994, pp. 487-499.

[2] M. J. A. Berry and G. S. Linoff, Mastering Data Mining, John Wiley \& sons, 2000.

[3] P. N. Tan, M. S. Bach, and V. Kumar, Introduction to Data Mining, Pearson Publications, 2009.

[4] P.-N. Tan et al., Introduction to Data Mining, Addison Wesley, 2006.

[5] J. W. Han and M. Kamber, Data Mining: Concepts and Techniques, Morgan Kaufmann Publishers, 2006.

[6] Y.Z. Zhang, "Spatial data cube: provides better support for spatial data mining, Geoscience and Remote Sensing Symposium," in Proc. IGARSS '05, IEEE International, 2005, pp. 795-798.

[7] B. Li, L. B. Shi, and S. P. Liu, "Research on spatial data mining based on uncertainty in government GIS," in Proc. Seventh International Conference on Fuzzy Systems and Knowledge Discovery, 2010, pp. 2095- 2908,

[8] R. R. Yager and F. E. Petry, "A Multicriteria approach to data summarization using concept ontologies," IEEE transaction on fuzzy systems, vol. 14, no. 6. pp. 769-780, 2006.

[9] W. Bandler and L. Kohout, "Fuzzy power sets and fuzzy implication operators," Fuzzy Sets Syst., vol. 4, pp. 13-30, 1980.

[10] S. Nassar and J. Sander, "Effective summarization of multi-dimensional data streams for historical stream mining, ssdbm," in Proc. 19th International Conference on Scientific and Statistical Database Management, pp. 30-40, 2007.

[11] P. Umamaheshwari and M. Rajaram "A novel approach for mining association rules on sports data using principle component analysis: for cricket match perspective," IEEE International Advance Computing Conference, Patiala, India, 6-7 March. pp. 1074-1080, 2009.

[12] J. W. Han, "Intelligent query answering by knowledge discovery techniques," IEEE Transaction on Knowledge and Data Engineering, vol. 8, no. 3, pp. 373-390, 1996.

[13] Q. Ding and G. Sundarraj, "Frequent pattern Discovery and Association Rule Mining from XML Data," in XML Data Mining: Models, Methods, and Applications, A. Tagarelli, ed., IGI Global, 2011, pp. 243-263.

[14] C. Turkay, A. J. Lundervold, and H. Hauser, "Representative factor generation for the interactive visual analysis of high- dimensional data," IEEE Transactions on Visualization and Computer Graphics, vol. 18 , no. 12 , pp. 2621-2631.

[15] D. J. Hand, "Deconstructing statistical questions (with discussion)," Journal of the Royal Statistical Society, vol. 57, pp. 317-356, 1994.

[16] P. Brazdil, J. Gama, and Henery, "Characterizing the Applicability of Classification Algorithms using Meta Level Learning," in Proc. ECML, vol. 784, 1994, pp. 83-102.

[17] S. Craw, D. Sleeman, N. Graner, M. Rissakis, and S. Sharma, "CONSULTANT: Providing advice for The Machine Learning Toolbox," M. Bramer, ed., in Proc. the 12th Annual Technical Conference of the British Computer Society Specialist Group on Expert Systems, Cambridge, UK. 1992, pp. 5-23.

[18] F. Verdenius, "Applications of inductive learning techniques: A survey Netherlands," AI Communications, vol. 10, no. 1, pp. 3-20, 1997. 
[19] C. Brodley and P. Smyth, "Applying Classification Algorithm in Practice," in Proc. the workshop on Applying Machine Learning in Practice at the ICML-95, 1995.

[20] W. Malcolm et al., "Interactive Machine Learning: Letting users build classifiers," J. of Human Computer Studies, vol. 55, no. 3, 2001.

[21] J. Hellersterin, R. Avnur, A. Chou, C. Hidber, V. Raman, T. Roth, and P. Hass, "Interactive Data analysis: The Control Project," IEEE Computer, vol. 32, no. 80, pp. 51-59, 1999

[22] R. Cooley, B. Mobasher, and J. Srivastava, "Web Mining: Information and Pattern Discovery on the world wide web (A survey paper)," in Proc. the $9^{\text {th }}$ IEEE International Conference on Tools with Artificial Intelligence, 1997.

[23] Q. J. Chen, X. D. Wu, and X. Q. Zhu, "Online Interactive Data Mining," in Proc. the 17th International Conference on Innovations in Applied Artificial Intelligence, 2004, pp. 66 - 76.

[24] H. K. Mohammed and A. F. Soliman, "Intelligent Query Answering with Data Mining Techniques, Computer Engineering \& Systems," International Conference on Communication, Networking \& Broadcasting, pp. 427-432.

[25] F. S. Gharehchopogh, "Approach and Review of User Oriented Interactive Data Mining," in Proc. the 4th International Conference on Application of Information and Communication Technologies (AICT2010), Digital Object Identifier: 10.1109/ICAICT.2010.5611792, IEEE, Tashkent, Uzbekistan, 2010, pp. 1-4.

[26] W. J. Li, F. Wang, and H. Deng, "A Survey on Automatic Summarization, Information Technology and Applications (IFITA)," in Proc. 2010 International Forum on Computing \& Processing (Hardware/Software), Kunming, 2010, pp. 193-196.

[27] A. d'Acierno, V. Moscato, and A. Penta, "iWIN: a Summarizer System Based on a Semantic Analysis of Web Documents, Semantic Computing (ICSC)," in Proc. 2012 IEEE Sixth International Conference on Communication, Networking \& Broadcasting; Components, Circuits, Devices \& Systems; Computing \& Processing (Hardware/Software), General Topics for Engineers (Math, Science \& Engineering, 2012, pp. 162-169.

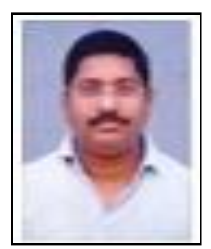

M. Manohar was brought up in Udupi and currently he lives in Bangalore, Karnataka. He was qualified Bachelor in CSE in the year 1999 at Dr AIT, Bangalore. He has his Master Degree in CSE in the year 2004 at D AIT, Bangalore. He is pursuing his Ph.D in CSE in the area of data mining and big data.

$\mathrm{He}$ is an assistant professor in the Information Science \& Engineering Department at School of Engineering \& Technology of Jain University, Bangalore. He is an educator by choice and vocation, with an experience of 13 years in Teaching. He is a member of Professional body IACSIT, IET.

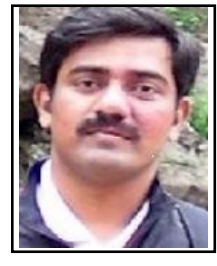

R. Dinesh comes from Mysore and currently he lives in Bangalore, Karnataka. He has completed M.Sc and $\mathrm{Ph} . \mathrm{D}$ in computer science both from the University of Mysore. His area of research includes, image processing, computer vision, pattern recognition, data analytics

Currently he is working as a Technical Manager at HCL Technologies, Bangalore. He has many publications and patents to his credits. He is also serving as a member of editorial boards in many journals. He is presently supervising many $\mathrm{Ph} . \mathrm{D}$ candidates

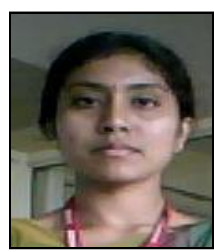

M. S. Sowmya was brought up in Mysore and currently he lives in Bangalore, Karnataka. She is qualified Bachelor in CSE in the year 2008 at GCE, Bangalore. She has her Master Degree in CSE in the year 2012 at RVCE, Bangalore. She is an Assistant Professor in the Information Science \& Engineering Department at School of Engineering \& Technology of Jain University, Bangalore. She is a passionate educator, having 5 years of experience in her Teaching career. She has received an appreciation from the College of St Aloysius, Mangalore, for training students on paper presentations. She is a member of Professional body IACSIT, MIET 